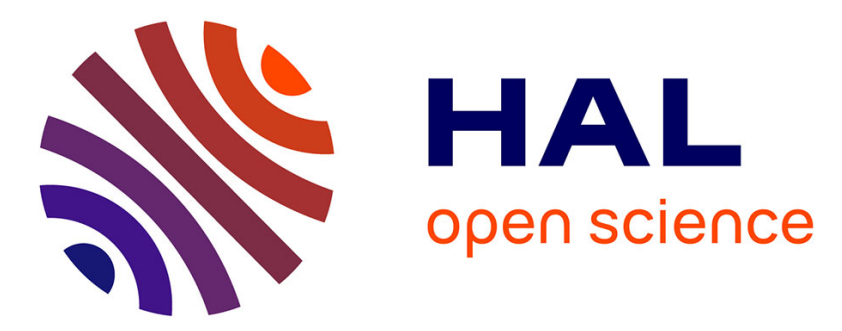

\title{
Toward cognitive radio resource management based on multi-agent systems for improvement of real-time application performance
}

Asma Amraoui, Fatima Zohra Benidris, Badr Benmammar, Francine Krief, Fethi Tarik Bendimerad

\section{To cite this version:}

Asma Amraoui, Fatima Zohra Benidris, Badr Benmammar, Francine Krief, Fethi Tarik Bendimerad. Toward cognitive radio resource management based on multi-agent systems for improvement of realtime application performance. Fifth IFIP International Conference on New Technologies, Mobility and Security (NTMS'2012), May 2012, Istanbul, Turkey. hal-00680109v2

\section{HAL Id: hal-00680109 \\ https://hal.inria.fr/hal-00680109v2}

Submitted on 18 Mar 2012

HAL is a multi-disciplinary open access archive for the deposit and dissemination of scientific research documents, whether they are published or not. The documents may come from teaching and research institutions in France or abroad, or from public or private research centers.
L'archive ouverte pluridisciplinaire HAL, est destinée au dépôt et à la diffusion de documents scientifiques de niveau recherche, publiés ou non, émanant des établissements d'enseignement et de recherche français ou étrangers, des laboratoires publics ou privés. 


\title{
Toward cognitive radio resource management based on multi-agent systems for improvement of real-time application performance
}

\author{
Asma Amraoui ${ }^{1}$, Fatima zohra Benidris ${ }^{1}$, Badr Benmammar ${ }^{1}$, Francine Krief $^{2}$, Fethi Tarik Bendimerad ${ }^{1}$ \\ ${ }^{1}$ LTT Laboratory of Telecommunication Tlemcen \\ UABT Tlemcen, Algerie \\ ${ }^{2}$ LaBRI Laboratory Bordeaux 1 University \\ 33400 Talence, France \\ \{amraoui.asma, fatima.benidriss, badr.benmammar, ftbendimerad\}@gmail.com, krief@labri.fr
}

\begin{abstract}
Cognitive Radio (CR) is a promising technology that can alleviate the spectrum shortage problem by enabling unlicensed users equipped with CRs to coexist with incumbent users in licensed spectrum bands while causing no interference to incumbent communications. In this paper, we propose a new approach which uses $\mathrm{CR}$ for improving real-time application performance related to only one cognitive radio mobile terminal (CRMT). We also present a synthesis of research relating to dynamic spectrum allocation and sharing in the context of CR networks using multi-agent systems. In our future work, we will seek to improve real-time application performance related to many CRMT.
\end{abstract}

Keywords: Cognitive radio, multi-agent systems, cognitive radio mobile terminal, handover, artificial intelligence.

\section{INTRODUCTION}

We currently attend the multiplication of telecommunication standards considering recent progress in this area. The increasing number of standards broadens the range of offers and available services for each user; however, most available radio frequencies have already been allocated.

A study carried out by the Federal Communications Commission (FCC) has shown that some frequency bands are overloaded at the rush hours. However, the use of the frequency spectrum is not uniform: according to the hours of day and to the geographical position; a frequency band can be overloaded while another remains unused. The idea to develop tools to better use the spectrum has naturally emerged.

Cognitive Radio (CR) is the concept that meets this challenge; better use the spectrum, it is also to increase the throughput and make more reliable the physical layer.

Most researches on CR networks have focused on the exploitation of unused spectrum. However, the CR nodes possess the necessary qualities to make a considerable progress in the reliability of wireless networks [1], which has been less explored, so that is why we were interested by improving wireless link reliability in order to improve realtime application performance, we are specially interested in video conferencing application.

The aim of our paper is to propose a technique to improve real-time application performance related to only one CRMT. Our technique is based on machine learning. In our future work, we will seek to improve real-time application performance related to many CRMT based on multi-agent systems.

This paper is organized as follows. First, we describe the case related to one CRMT using video conferencing application and the results of our experimentation. Then, we present a synthesis of research relating to dynamic spectrum allocation and sharing in the context of CR networks using multi-agent systems. Our objective is to improve real-time application performance related to many CRMT using multi-agent systems.

\section{SCENARIOS AND PROPOSED SOLUTIONS RELATED TO ONLY ONE CRMT}

\section{A. Scenario}

The Figure 1 below shows a path followed by a CRMT when it switches to an area where the signal quality drops to an unacceptable level (shown in red) due to a gap in coverage, we assume that the client uses video conferencing over the route.

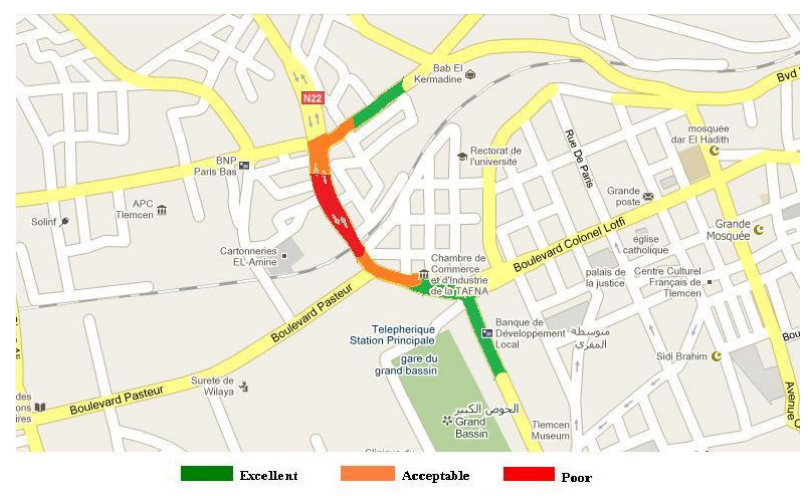

Figure 1. Signal quality associated to a cognitive radio

\section{B. Proposed Solution}

After several incidents, the CR should be aware of the problem. Then, through some geolocations or the ability to learn the time of the day when this happens, the radio can anticipate the difference in coverage and know the necessary signal to the base station to change characteristics of the signals when the user approaches the deficient coverage.

\section{Application}

As mentioned above, we will use video conferencing (real-time application) in the case of a mobile user who needs to take a path where the signal quality drops to an 
unacceptable level due to a gap in coverage, giving a very low QoS. This can be remedied by using the CR, but problematic arises: WHEN and WHY to use cognitive radio?

Most research related to the QoS of video conferencing, take into account throughput as pertinent parameter. For this reason, we choose the "Throughput" as a single pertinent parameter for our application. For this, a throughput classification is required, and as we play the role of the expert, we have created our own database following certain rules in order to apply our approach. The database was divided into two parts, the first one for learning and the second one for testing. The value of the throughput will change almost every time it is measured during the day even on the same route, for this reason, our measures have been taken into account for 5 weeks at 3 different intervals of the day (8am - 11am, 11am - 3pm, 3pm - 5pm) excluding weekend.

However, as we don't have real data used in the CR and it is the case of the whole community, we had to play the role of the expert to assign the needed data for our simulation. In the literature, we found that to have a good QoS in video conferencing, throughput must be $>384 \mathrm{~Kb} / \mathrm{s}$.

We proposed to affect throughput on three classes:

- Gold for samples where the throughput is greater than $384 \mathrm{~Kb} / \mathrm{s}$, ensuring $100 \%$ quality satisfaction of the user.

- $\quad$ Silver for samples where the throughput is between $160 \mathrm{~Kb} / \mathrm{s}$ and $384 \mathrm{~Kb} / \mathrm{s}$, of acceptable quality.

- Bronze for samples where the throughput is less than $160 \mathrm{~Kb} / \mathrm{s}$. This means that video conferencing is not satisfactory, and it is the class that interests us because this is when we use the CR.

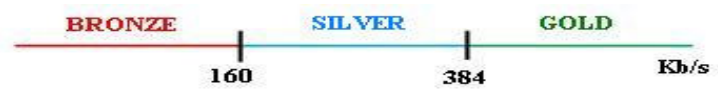

Figure 2. Database classes

\section{C.1 First question "WHEN"?}

For the data classification, we used three different algorithms derived from the field of machine learning.

- $\quad$ The k-nearest neighbor's algorithm (K-NN) which is a supervised classification algorithm.

- The multilayer perceptron algorithm (MLP of neural networks).

- The C4.5 algorithm of decision trees.

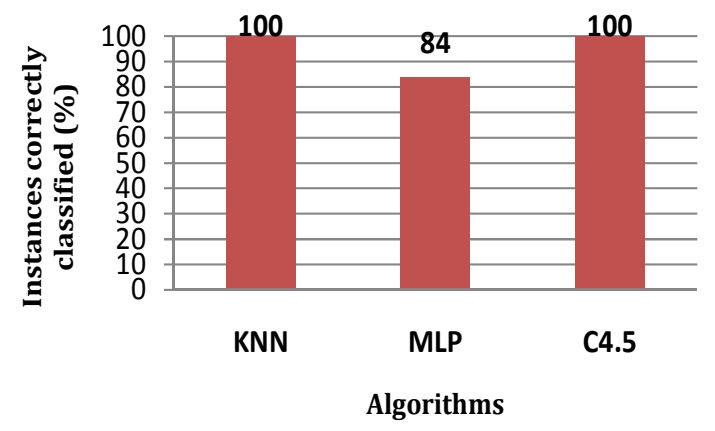

Figure 3. Best scores obtained with the three algorithms
We remark that, the multi layer perceptron has not given satisfactory results compared with the other algorithms; however we did not get $100 \%$, despite the change of parameters such as the number of hidden layers, the number of epochs and the learning rate.

We remark also that the two other algorithms (KNN and C4.5) have given results of $100 \%$. This is why we chose the $\mathrm{K}-\mathrm{NN}$ algorithm and focus on the obtained results.

This algorithm (K-NN) was tested with several values of $\mathrm{K}$ on the test database and each time the result was different, but it is satisfactory until the value of $K=6$.

TABLE I. ClASSIFICATION OBTAINED WITH KNN

\begin{tabular}{|l|l|l|l|l|}
\hline $\begin{array}{c}\text { Values of } \\
\mathbf{K}\end{array}$ & \multicolumn{2}{|c|}{$\begin{array}{c}\text { Instances correctly } \\
\text { classified }\end{array}$} & \multicolumn{2}{c|}{$\begin{array}{c}\text { Misclassified } \\
\text { instances }\end{array}$} \\
\hline $\mathrm{K}=1$ & 20 & $100 \%$ & 0 & $0 \%$ \\
\hline $\mathrm{K}=2$ & 19 & $95 \%$ & 1 & $5 \%$ \\
\hline $\mathrm{K}=3$ & 19 & $95 \%$ & 1 & $5 \%$ \\
\hline $\mathrm{K}=4$ & 18 & $90 \%$ & 2 & $10 \%$ \\
\hline $\mathrm{K}=5$ & 18 & $90 \%$ & 2 & $10 \%$ \\
\hline $\mathrm{K}=6$ & 18 & $90 \%$ & 2 & $10 \%$ \\
\hline
\end{tabular}

For $\mathrm{K}=1$ : All the throughput examples were well posted in their appropriate class.

Whereas, with $\mathrm{K}=2$, one sample was misclassified, we notice that in the graph.

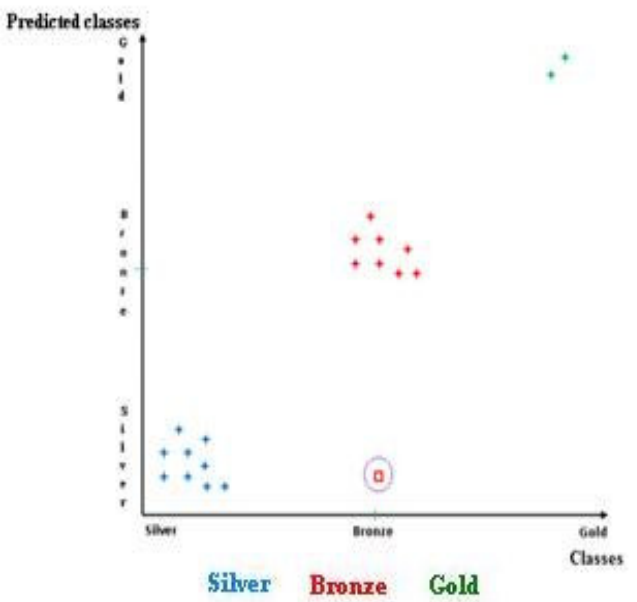

Report: according to the results of the classification, the cognitive radio will be activated each first day of week from $8 \mathrm{am}$ to $11 \mathrm{am}$ and from $3 \mathrm{pm}$ to $5 \mathrm{pm}$, and every Wednesday from 8 am to $5 \mathrm{pm}$ because in these intervals the throughput belongs to the Bronze class $<160 \mathrm{~Kb} / \mathrm{s}$. So, through this, the question When is answered.

\section{C.2 Second question "WHY"?}

Now, We will justify the usefulness of the CR, this by supposing that the spectrum sensing is already done by the receiver of our mobile terminal which is in this case a multimode wireless communication terminal (MWCT), so capable to support multiple access technologies such as GSM, UMTS or WiMAX.

Considering that, the spectrum is not used at $100 \%$, we can represent the frequency bands into two sets: the first contains the occupied bands and the second contains the free ones. 
The CRMT must switch to an unused frequency band among those available in the free bands set.

For our approach, scenarios were studied to show the usefulness of the CR, this based on the number of free bands and the time of use of each one, we identified three possible scenarios:

- Favorable (best case): the CRMT receiver detects a free band and uses it during all the way without any interruption caused by the primary user.

- Unfavorable (worst case): the CRMT receiver does not detect any free band (empty set) or it detects some bands but their use interferes with the primary users. In this case the CR is not used because the secondary user should not disturb the primary users.

- Common ( $\mathrm{N}$ frequency with $\mathrm{N}$ hops): the terminal uses a free band b1, then there is an interruption caused by the primary user, so it switches to another free band b2 (he made a hop). If the primary user of $\mathrm{b} 2$ needs his band, the secondary user must switch again, and so on, until the end of the condition when he opts for the CR (before returning to his initial frequency band), he will have done $\mathrm{N}$ hops.

For our application, we have based on the number of hops done by the CR during all the way of the secondary user. In each of the scenarios mentioned above we calculated the time of interruption which is the required time for the terminal to access a free band and use it.

The time of interruption will be defined as follows: T.interruption $=(T$.sensing $+\mathrm{T}$. establishment $) \mathrm{x}$ number of hops.

The sensing time is the required time to detect a free band, it is negligible compared to the time of establishment, it is even included in the time of establishment for some algorithms which treat the diagonal handover (switching between wireless networks which uses the subjacent technologies such as the standard IEEE 802 ) or the vertical handover (switching from one access technology to another).

In the literature, we found that the necessary time for establishment to exploit a free frequency band of another technology is $\mathbf{5}$ seconds on average [2] and [3].

T.sensing $<<$ T.establishment, which gives us:

T.interruption $=\mathrm{T}$. establishment $\mathrm{x}$ number of hops. We call time of rupture: the necessary time to return to the initial frequency band. So, it is the sum of the time of interruption and the time of use of each band, knowing that, the time of use can differ from a band to another according to the primary user.

T.rupture $=$ T.interruption + T.use

\section{C.3 Experimentation results}

To support our proposal and to better understand, we compared the QoS with and without cognitive radio (case without CR means that our terminal has only one access technology and works on the same frequency band). The graphs below illustrate this comparison. For being readable, we supposed that the maximum time of rupture is $5 \mathrm{~min}$ and that the free band set contains 7 bands.

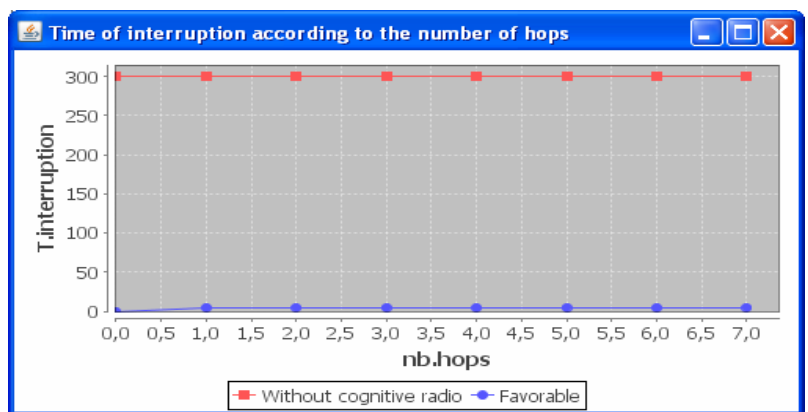

Figure 7. Comparison between the favorable scenario and the case without cognitive radio

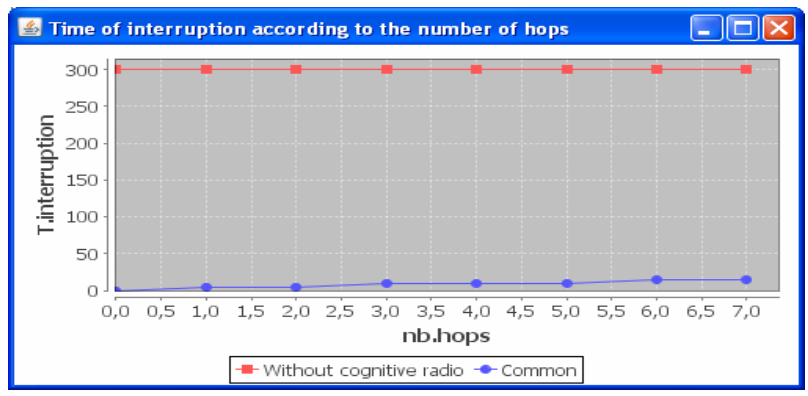

Figure 8. Comparison between the common scenario and the case without cognitive radio

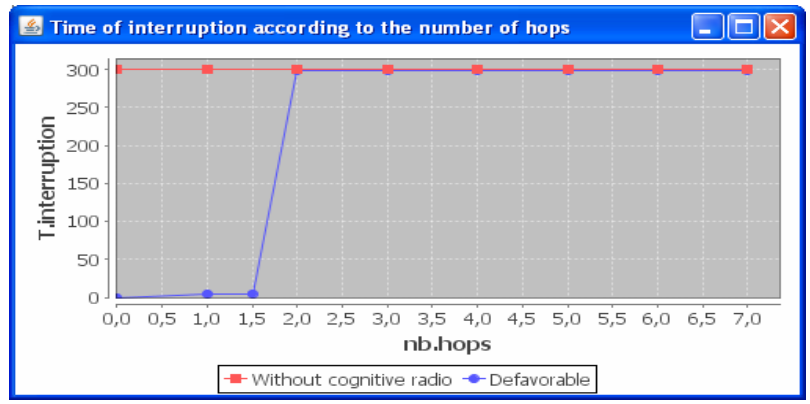

Figure 9. Comparison between the defavorable scenario and the case without cognitive radio

\section{C.4 Results interpretation}

Modeled graphs above represent the downtime depending on the number of hops performed. We note that whatever the number of hops, the case with the CR is much better than that without CR. According to the first graph, of course we lost 5 seconds to connect to the new band but we see that the CR has saved us 295 s which is the time without interruption. For the second graph, we performed three hops, which mean that the downtime is 5s x 3 hops: 15s. Despite this, the case with CR is always better. On the third graph, a single hop was made but the connection was interrupted by the primary user at the beginning, so we took advantage of the $\mathrm{CR}$ for a few seconds.

Result: whatever the number of hops performed, the use of $\mathrm{CR}$ is always more efficient.

In summary, we can say that $\mathrm{CR}$ is an efficient tool to improve real-time application performance related to only one CRMT. 


\section{MULTI-AGENT SYSTEMS FOR DYNAMIC SPECTRUM ALLOCATION AN SHARING IN THE CONTEXT OF CR NETWORKS}

Very few researchers have applied multi-agent systems for dynamic spectrum allocation and sharing in the context of CR networks.

In [4], the author proposes a muti-agent systems based solutions to achieve licensed and unlicensed dynamic spectrum sharing. Firstly, they present a cooperative approach where the $\mathrm{CR}$ nodes embarked with agents are capable of performing spectrum sharing by exchanging a series of messages with the neighboring licensed devices. While analyzing the performance of this proposal under ad-hoc wireless conditions, they show that it achieves good performance in term of spectrum access, without incurring greater communication cost. Then, they focus on enabling unlicensed spectrum sharing between the CR users. Their proposed solutions can achieve good performance while maintaining fair spectrum distribution.

A muti-agent systems based spectrum sharing strategy is proposed in [5], specifically, a cooperative muti-agent systems is considered, in which the agents are deployed over primary and secondary user devices. The developed cooperation mechanism is similar to that of contract net protocol (CNP), in which the individual secondary user (SU) agent should send messages to the appropriate neighboring primary user (PU) agents whenever needed and, subsequently, the related PU agents should reply to these agents in order to make a spectrum sharing agreement. The paper proposes that the SU agents should take their decisions based on the amount of spectrum, time and price proposed by the PU agents and should start spectrum sharing whenever they find an appropriate offer (without waiting until the reception of all the neighboring PU agents' responses). Then, after completely utilizing the desired spectrum, SU agents should pay the agreed price to the respected PU agents.

In [6], the paper investigates cooperative radio resource management for multiple cognitive radio networks in interference environments. The objective of this research is to manage shared radio resources fairly among multiple non-cooperative cognitive radio networks to optimize the overall performance. A multiagent-system-based approach is proposed to achieve information sharing and decision distribution among multiple cognitive radio networks in a distributed manner.

The paper [7] proposes a multi-agent learning algorithm and applies it for optimizing online resource allocation in cluster networks. The learning is distributed to each cluster, using local information only and without access to the global system reward.

In [8], a novel intelligent multi-agent radio resource management system is proposed, which is self-organized and distributed to ensure the coexistence of multi-radio access technologies. Radio resource is managed by a macro control and management system using control factors and validation mechanism, instead of micro control for individual users. The goal is to increase radio resource utilization efficiency, maximize system capacity and meet the QoS requirements of different services.

The work done in [9] proposes muti-agent systems that adopt mobile agents as a technology for tasks distribution, results collection, and management of resources in largescale distributed systems. A new mobile agent-based approach for collecting results from distributed system elements is presented. The technique of artificial intelligence based on intelligent agents giving the system a proactive behavior. As mentioned above, we can use multiagent systems for dynamic spectrum allocation and sharing in the context of CR networks.

\section{CONCLUSION}

We presented in this paper a new approach that uses cognitive radio to improve real-time application performance for a cognitive radio mobile terminal. In our future work, we think we can improve real-time application performance related to many CRMT [10] [11] [12] [13] [14] using multi-agent systems.

\section{REFERENCES}

[1] J. Neel, "Analysis and Design of Cognitive Radio Networks and Distributed Radio Resource Management Algorithms", Faculty of the Virginia Polytechnic Institute and State University, September 2006.

[2] S. Busanelli, M. Martalõ, G. Ferrari, and G. Spigoni, "Vertical Handover between $\mathrm{WiFi}$ and UMTS Networks: Experimental Performance Analysis", International Journal of Energy, Information and Communications Vol. 2, Issue 1, February 2011.

[3] Z. Daia, R. Fracchiaa, J. Gosteaub, P. Pellatia, G. Viviera, "Vertical handover criteria and algorithm in IEEE 802.11 and 802.16 hybrid networks", Laboratoire de Motorola Paris. International Conference On Communications (ICC 2008).

[4] U. Mir, L. Merghem-Boulahia, M. Esseghir and D. Gaïti "Dynamic Spectrum Sharing for Cognitive Radio Networks using Multi-agent System" The 8th Annual IEEE Consumer Communications and Networking Conference (CCNC), 2011.

[5] U. Mir, L. Merghem-Boulahia, and D. Gaïti, "A cooperative multiagent based spectrum sharing", in Proc. 6th Annu. Advanced International Conference on Telecommunications, Barcelona, 2010.

[6] X. Jiang, H. Ivan, and R. Anita, "Cognitive radio resource management using multi-agent systems" in Proc. 4th Annu. IEEE International Conf. Consumer Communications and Networking, Vegas, 2007, pp. 1123-27.

[7] C. Zhang, V. Lesser, and P. Shenoy, "A multi-agent learning approach to online distributed resource allocation" Proc. International Joint Conference on Artificial Intelligence (IJCAI 09), 2009.

[8] Feng, Z; Yu, K; Ji, Y; Zhang, P; Li, VOK; Zhang, Y "Multi-access Radio Resource Management using Multi-Agent System“. IEEE Wireless Communications and Networking Conference, Las Vegas, Nevada, USA, 3-6 April 2006, v.1, p. 63-68, 2006.

[9] H. M. Kelash, H. M. Faheem, and M. Amoon, "A Multi-agent System for Distributed Systems Management", proceedings of world academy of science, engineering and technology volume 11 februry 2006 ISSN 1307-6884.

[10] A. Amraoui, W. Baghli, B. Benmammar, "Improving video conferencing application quality for a mobile terminal through cognitive radio", 14th IEEE International Conference on Communication Technology (ICCT 2012). Chengdu, China, November 9th-11th, 2012. to appear.

[11] A. Amraoui, W. Baghli, B. Benmammar, "Amélioration de la fiabilité du lien sans fil pour un terminal radio cognitive mobile", 12 ème Journées Doctorales en Informatique et Réseau (JDIR'11), Belfort, France, Pages : 1-6, 2011.

[12] B. Benmammar, A. Amraoui and W. Baghli, "Performance improvement of wireless link reliability in the context of cognitive radio". IJCSNS International Journal of Computer Science and Network Security, Vol.12, No.1, Pages: 15-22, 2012.

[13] N. Samaan, B. Benmammar, F. Krief and A. Karmouch. "Prediction-based Advanced Resource Reservation in a Mobile Environment". 18th IEEE Annual Canadian Conference on Electrical and Computer Engineering, CCECE05, May 1-4, 2005, Saskatoon Inn, Saskatoon, Saskatchewan Canada.

[14] B. Benmammar and F. Krief. "MQoS NSLP: a mobility profile management based approach for advance resource reservation in a mobile environment". Proceedings of the 7th IFIP IEEE International Conference on Mobile and Wireless Communications Networks (MWCN 2005). Marrakech, Morocco. September 19-21, 2005. 